\title{
BMJ Open Neurological complications of breast cancer: study protocol of a prospective cohort study
}

\author{
Susana Pereira, ${ }^{1,2,3}$ Filipa Fontes, ${ }^{2,3}$ Teresa Sonin, ${ }^{1}$ Teresa Dias, ${ }^{1}$ Maria Fragoso, ${ }^{1}$ \\ José Castro-Lopes, ${ }^{4,5}$ Nuno Lunet ${ }^{2,3}$
}

To cite: Pereira S, Fontes F, Sonin T, et al. Neurological complications of breast cancer: study protocol of a prospective cohort study. BMJ Open 2014;4:e006301. doi:10.1136/bmjopen-2014006301

- Prepublication history for this paper is available online. To view these files please visit the journal online (http://dx.doi.org/10.1136/ bmjopen-2014-006301).

Received 4 August 2014 Accepted 22 August 2014

CrossMark

\footnotetext{
${ }^{1}$ Instituto Português de Oncologia, Porto, Portugal ${ }^{2}$ EPI Unit, Instituto de Saúde Pública da Universidade do Porto (ISPUP), Porto, Portugal

${ }^{3}$ Departamento de Epidemiologia Clínica, Medicina Preditiva e Saúde Pública, Faculdade de Medicina, Universidade do Porto, Porto, Portugal ${ }^{4}$ Departamento de Biologia Experimental, Faculdade de Medicina, Universidade do Porto, Porto, Portugal ${ }^{5}$ Instituto de Biologia Molecular e Celular (IBMC), Universidade do Porto, Porto, Portugal
}

Correspondence to Dr Nuno Lunet; nlunet@med.up.pt

\section{ABSTRACT}

Introduction: The improvement in breast cancer survival rates, along with the expected overdiagnosis and overtreatment associated with breast cancer screening, requires a comprehensive assessment of its burden. Neurological complications can have a devastating impact on these patients; neuropathic pain and chemotherapy-induced peripheral neuropathy are among the most frequently reported. This project aims to understand the burden of neurological complications of breast cancer treatment in Northern Portugal, and their role as mediator of the impact of the treatment in different dimensions of the patients' quality of life.

Methods and analysis: A prospective cohort study was designed to include 500 patients with breast cancer, to be followed for 3 years. The patients were recruited at the Portuguese Oncology Institute of Porto and evaluations were planned at different stages: pretreatment, after surgery, after chemotherapy (whenever applicable) and at 1 and 3 years after enrolment. Patients diagnosed with neuropathic pain or chemotherapy-induced peripheral neuropathy (subcohorts), were also evaluated at the moment of confirmation of clinical diagnosis of the neurological complication and 6 months later. In each of the followup periods, a neurological examination has been performed by a neurologist. Data were collected on sociodemographic and clinical characteristics, quality of life, sleep quality, and anxiety and depression. Between January and December 2012, we recruited and conducted the baseline evaluation of 506 participants. The end of the follow-up period is scheduled for December 2015.

Ethics and dissemination: The study protocol was approved by the Ethics Committee of the Portuguese Oncology Institute of Porto and all patients provided written informed consent. All study procedures were developed in order to assure data protection and confidentiality. Results from this project will be disseminated in international peer-reviewed journals and presented in relevant conferences.

\section{INTRODUCTION}

Breast cancer is the most frequent form of cancer and an important cause of cancer death among women, with an estimated 1.7

\section{Strengths and limitations of this study}

- This protocol describes an ongoing prospective cohort study with baseline evaluation already performed.

- The study was approved by the ethics committee of the hospital where the patients were recruited.

- The results of this study will be submitted for publication in international peer-reviewed journals.

- The expected results may contribute to a better understanding of the burden of neurological complications of breast cancer treatment and their role as mediators of the impact of the treatment in different dimensions of the patients' quality of life.

million new cases and half a million deaths worldwide. ${ }^{1}$ Despite upward trends in incidence rates, due to an increasing exposure to risk factors and widespread use of mammography screening, ${ }^{2}$ mortality has been declining in most affluent settings, ${ }^{3}$ reflecting improvements in access to earlier diagnosis and effective treatments. ${ }^{4} 5$ In Northern Portugal, the number of cases is expected to be nearly $50 \%$ higher in $2020,{ }^{6}$ assuming the most recent trends remain, and mortality rates have been declining since the 1990s in several regions. ${ }^{7}$

The improvement in breast cancer survival, ${ }^{8}$ along with the expected overdiagnosis and overtreatment associated with breast cancer screening, ${ }^{9}$ requires a comprehensive assessment of the burden of cancer, accounting for disability and losses in quality of life (QoL) due to the disease, treatment and sequelae. ${ }^{10}$ Although health-related QoL in women with breast cancer has been addressed in several studies, ${ }^{11-13}$ little attention has been dedicated to understanding the role of specific physical and psychological adverse effects of cancer management ${ }^{14-17}$ in different dimensions of the patients' QoL. 
Neurological complications of breast cancer treatment, including cognitive impairment, chemotherapyinduced peripheral neuropathy (CIPN), neuropathic pain (NP), encephalopathy and stroke, ${ }^{18} 19$ may cause symptoms more disabling than the cancer itself ${ }^{18}$; CIPN and NP are among the most frequently reported. ${ }^{18} 2021$

CIPN is a dose-limiting side effect of many chemotherapeutic agents that may lead to dose reduction and/or discontinuation of treatment. ${ }^{22}$ The incidence of CIPN depends on chemotherapy regimens, ${ }^{22}$ but the role of conditions such as diabetes or alcohol consumption have seldom been addressed. ${ }^{23-25}$ Chronic NP is estimated to affect over a third of treated patients, ${ }^{20} 21$ especially younger ones. ${ }^{26-29}$ Despite some studies addressing the relationship between quality of sleep, ${ }^{30} 31$ anxiety and depression ${ }^{32}$ and the occurrence of pain, there is little information on the impact of these factors, specifically in NP. Moreover, data on type of surgery ${ }^{26} 29$ and radiotherapy ${ }^{28} 2933$ as risk factors for NP are conflicting.

Although QoL is known to be impaired by pain, ${ }^{34} 35$ to our knowledge no previous studies addressed the role of NP or CIPN as mediators of the effect of breast cancer treatment in different dimensions of QoL.

The burden of neurological complications in women with breast cancer, including NP and CIPN, remains poorly understood, namely regarding their aetiology, frequency and impact on patients' QoL. Prospective studies providing a comprehensive characterisation of these frequent side effects, and a methodologically sound assessment of their determinants and associations with specific dimensions of QoL, may contribute to a more accurate characterisation of the burden associated with breast cancer in different settings, as well as help to develop strategies to minimise the impact of these conditions during treatment.

This project aims to understand the burden of neurological complications of breast cancer treatment and their role as mediators of the impact of the treatment in different dimensions of the patients' QoL in Northern Portugal. The main specific objectives are as follows:

1. To estimate the incidence of neurological complications during the first 3 years after the diagnosis of breast cancer, and to characterise the clinical features and management of NP and CIPN.

2. To quantify the relationship between factors such as type of treatment, depression, anxiety and sleep disturbance or diabetes and alcohol consumption and the occurrence of NP and CIPN;

3. To assess the role of NP and CIPN as determinants of the variation in different dimensions of the patients' QoL.

\section{METHODS AND ANALYSIS}

Study design

This prospective cohort study was designed to evaluate a cohort of 500 women with incident breast cancer (main cohort) and subcohorts of patients diagnosed with NP
(NP subcohort) and CIPN (CIPN subcohort), during a 3-year follow-up period (figure 1).

The study comprises the evaluation of all participants at baseline (before any treatment), 2 weeks after surgery, 3 weeks after chemotherapy (if applicable) and at 1 and 3 years after enrolment. In addition, the subcohorts of patients are evaluated at the moment of confirmation of clinical diagnosis of the neurological complication and 6 -months after the diagnosis of the side effect (figure 1), in order to evaluate the chronicity of such conditions. The evaluations are performed by trained interviewers or clinicians, as applicable.

\section{Selection of participants}

Women admitted to the Breast Clinic of the Portuguese Institute of Oncology of Porto (IPO-Porto) suspected of having an incident breast cancer were potentially eligible. In 2012, we invited those who were proposed for surgery, either as primary treatment or after neoadjuvant therapy, aged 18 years or older, with histologically confirmed breast cancer diagnosed in the previous 3 months, not treated with chemotherapy and/or radiotherapy for other primary cancer, not having received any treatment for breast cancer before, not submitted to a previous breast surgery and capable of understanding the purposes of the study and willing to collaborate. We excluded those expected to receive cancer treatments other than surgery, if applicable, outside IPO-Porto.

We evaluated the cognitive function of each patient who accepted the invitation to participate, using the Montreal Cognitive Assessment. ${ }^{36}$ Those scoring less than 17 , or less than 16 for women over 65 years old, ${ }^{37}$ were excluded from further evaluation.

\section{Study questionnaires}

Table 1 depicts the questionnaires used to evaluate the participants at baseline and at different stages of follow-up, and table 2 describes the instruments validated for the Portuguese population, which were used to assess cognitive function, ${ }^{36} \mathrm{QoL}^{39-42}$ quality of sleep, ${ }^{43}{ }^{44}$ anxiety and depression, ${ }^{45} 46 \mathrm{NP},{ }^{47} 48$ pain severity ${ }^{48} 49$ and pain-related disability. ${ }^{48} 50$

\section{Neurological evaluation}

Newly occurring cases of neurological complications are identified through referral by any member of the clinical team, or during the systematic neurological evaluations described in table 1 . Prevalent cases identified at the time of the scheduled evaluations are assigned an estimated date of onset based on information provided by the patients.

The systematic neurological evaluation, performed by a neurologist, comprises the assessment of cognitive functions, cranial nerves, muscular strength, sensitive function, reflexes, Babinski signal and evaluation of gait and coordination. 


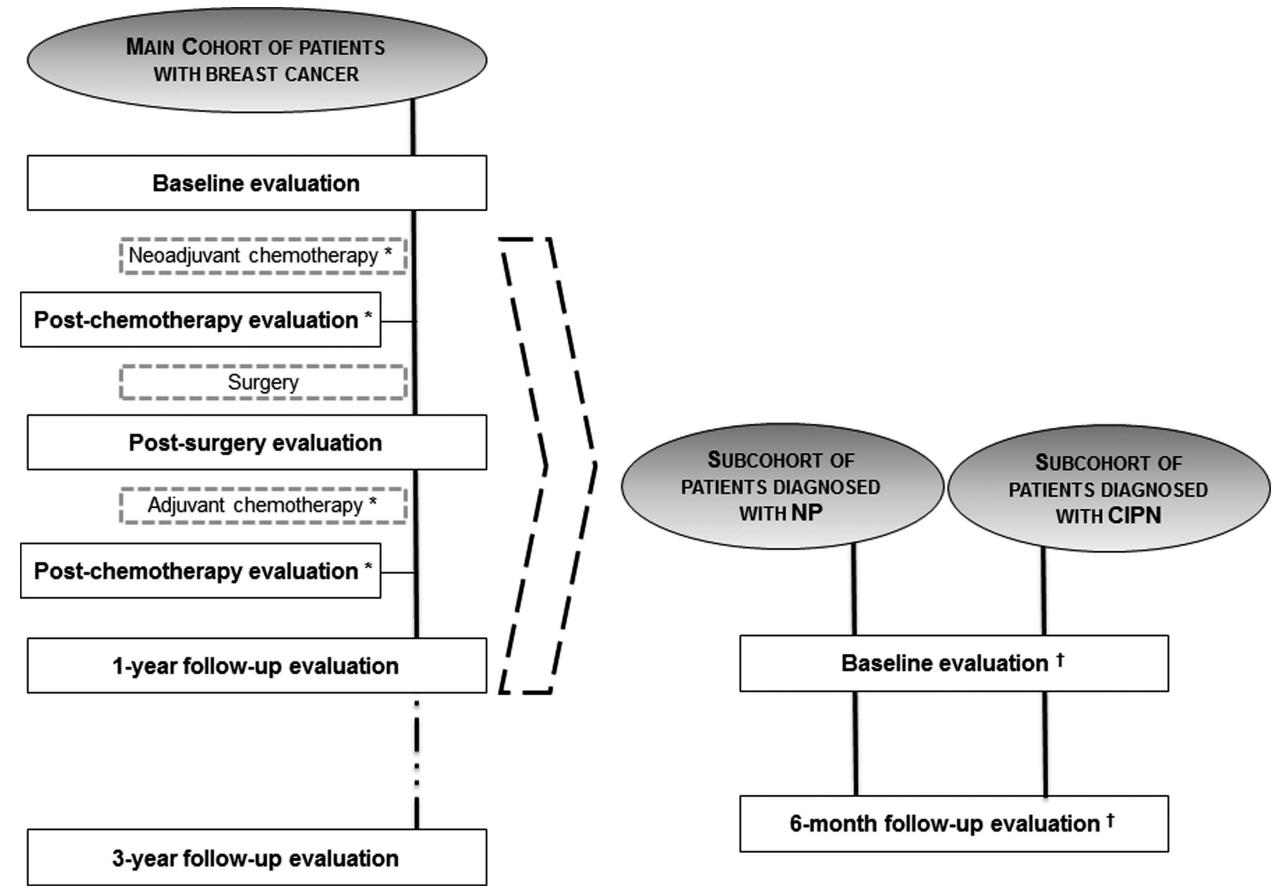

Figure 1 Study design and timing of baseline and follow-up evaluations in the main cohort and neuropathic pain and chemotherapy-induced peripheral neuropathy subcohorts. CIPN, chemotherapy-induced peripheral neuropathy; NP, neuropathic pain. *Not all patients are eligible for chemotherapy; †In addition to the evaluations that are performed for the main cohort.

\section{Data analysis and sample size}

We will compute cumulative incidence estimates and the corresponding 95\% CIs for each of the neurological complications at 6, 12 and 36 months of follow-up. A sample of about 500 participants is needed to estimate cumulative incidences between 30 and $70 \%$ with a $95 \%$ CI up to $10 \%$ wide, or cumulative incidences near or under $30 \%$ with a $95 \%$ CI up to $8 \%$ wide. We will conduct descriptive analyses to characterise NP and CIPN regarding their clinical features and management among the patients included in the corresponding subcohorts.

To quantify the association between different factors and the occurrence of NP and CIPN, we will compute incidence rate ratios and 95\% CI estimates, crude and adjusted for sociodemographic, clinical and QoL variables, using Poisson regression. A sample of approximately 500 women was estimated to be necessary, assuming a statistical power of $80 \%$, a level of significance of $5 \%$ and: (1) one-third of the sample exposed to each of the risk factors evaluated (eg, mastectomy; anxiety and/or depression; poor sleep quality), an incidence rate of NP of at least 30/100 person-years in the first year and a relative risk estimate of at least 1.5; and (2) approximately half of the women submitted to chemotherapy, $10 \%$ of the sample exposed to each of the risk factors evaluated (eg, diabetes; high alcohol consumption), an incidence rate of CIPN of at least 20/100 person-years in the first year and a relative risk estimate of at least 2.

The association between NP and CIPN and the variation in QoL from baseline to 1-year evaluation and from 1-year to 3-year follow-up assessments will be quantified through crude and adjusted incidence rate ratios and 95\% CI estimates, using Poisson regression. A sample of approximately 500 women was estimated to be necessary, assuming a statistical power of $80 \%$, a level of significance of $5 \%$, one-third of the sample with incident NP, an incidence rate of 25/100 person-years for moderate clinically meaningful worsening in QoL (decrease of at least 10 points in the Quality of Life Questionnaire of the European Organization for Research and Treatment of Cancer (QLQ-C30) score ${ }^{53}$ ) and a relative risk estimate of at least 1.5. A sample of approximately 200 women was estimated to be necessary, assuming a statistical power of $80 \%$, a level of significance of $5 \%$, one-fifth of the sample with incident CIPN, an incidence rate of 25/100 personyears for moderate clinically meaningful worsening in QoL (decrease of at least 10 points in the QLQ-C30 score $^{53}$ ) and a relative risk estimate of at least 2.

Training of the interviewers and use of standardised procedures for data collection is expected to contribute to a low proportion of missing data, and no imputation is being planned.

We estimate that the 3-year evaluation will be accomplished for at least $90 \%$ of the participants, taking into account the most recent survival data from Northern Portugal $^{54}$ and the fact that all women in our cohort were submitted to surgical treatment. The evaluations will be matched with routine appointments in the hospital, which is expected to contribute to minimise further loss to follow-up. 
Table 1 Description of methods used for evaluation of participants at baseline and at different stages of follow-up

Methods used for evaluation of participants

\section{Sociodemographic and Neurological}

Timing of evaluation clinical characteristics evaluation

\section{MoCA}

HADS

PSQI QLQ-BR23

QLQ-C30

BPI

DN4

Main cohort of patients with breast cancer

Baseline

Postsurgery

Postchemotherapy

1-year follow-up

3-year follow-up

Subcohort of patients diagnosed with NPt†

Baseline

6-month follow-up

\begin{tabular}{|c|}
\hline $\begin{array}{l}\boldsymbol{V}^{*} \\
\sqrt{ } \dagger \\
\sqrt{ } \\
\sqrt{ } \S \\
\sqrt{ }\end{array}$ \\
\hline
\end{tabular}

2
2
2
2
2

Subcohort of patients diagnosed with

$\checkmark \ddagger \ddagger$

CIPN††

Baseline

6-month follow-up

\section{$\checkmark$}

$\checkmark \S \S$

$\checkmark \checkmark \checkmark$

$\checkmark \quad \checkmark$

$\checkmark$

$\checkmark$

disease, diabetes, hypertension, thyroid pathology and oncological history) characteristics.

tData is collected on type of surgery, cancer stage ${ }^{51}$ and proposed treatment after surgery

†Data is collected on chemotherapy (drugs used, duration of treatment and total dose).

$\S$ Data is collected on radiotherapy (irradiated areas, total dose and duration of treatment) and hormonotherapy (drug), and other data is updated (marital status, cancer stage ${ }^{51}$ and information regarding chemotherapy and radiotherapy).

IData is collected on smoking habits, fruits and vegetables consumption, and physical activity. Marital status, alcohol consumption and information regarding cancer stage and treatment are reviewed.

${ }_{\star \star *}^{*}$ Applicable only when NP is present at the moment of evaluation.

${ }^{* *}$ Applicable only when NP is present at the moment of evaluation.
+ In addition to the evaluations that are performed in the main cohort.

$\dagger+$ In addition to the evaluations that are performed in the main cohort.
$\ddagger \neq D$ ata is collected concerning NP symptoms, aetiology, duration, localisation and pain management.

$\S \S$ Data is collected regarding CIPN symptoms and chemotherapy details; CIPN is graded using Common Terminology Criteria for Adverse Events V.4.0 $0^{52}$ and Total Neuropathy score. ${ }^{24}$

BPI, Brief Pain Inventory ${ }^{48} 49$; CIPN, chemotherapy-induced peripheral neuropathy; DN4, Neuropathic Pain Questionnaire 47 48; HADS, Hospital Anxiety and Depression Scale ${ }^{45}$ 46; MoCA, The

Montreal Cognitive Assessment ${ }^{36} 38$; NP, neuropathic pain; PDI, Pain Disability Index ${ }^{48} 50$; PSQI, Pittsburgh Sleep Quality Index ${ }^{43} 44$; QLQ-BR23, Breast cancer-specific module of the Quality

of Life Questionnaire of the European Organization for Research and Treatment of Cancer ${ }^{39}{ }^{40}$; QLQ-C30, Quality of Life Questionnaire of the European Organization for Research and

Treatment of Cancer ${ }^{39}$

Treatment of Cancer ${ }^{3}$ di 
Table 2 Description of the instruments used for evaluation of the participants

\begin{tabular}{|c|c|c|c|}
\hline Instruments & Description & Domains/subscales & Score \\
\hline $\mathrm{MoCA}^{3638}$ & $\begin{array}{l}\text { Test for the rapid screening of } \\
\text { mild cognitive impairment-an } \\
\text { intermediate clinical state } \\
\text { between normal cognitive aging } \\
\text { and dementia }\end{array}$ & $\begin{array}{l}\text { Attention and concentration; } \\
\text { executive functions; memory; } \\
\text { language; visuoconstructional skills; } \\
\text { calculations; orientation }\end{array}$ & $\begin{array}{l}\text { Range: } 0-30 \\
\text { Higher scores represent better } \\
\text { cognitive performance }\end{array}$ \\
\hline $\mathrm{HADS}^{45} 46$ & $\begin{array}{l}\text { Scale with } 14 \text { questions } \\
\text { assessing anxiety and emotional } \\
\text { distress among patients during } \\
\text { the previous week }\end{array}$ & Depression; anxiety & $\begin{array}{l}\text { Range (for each subscale): } 0-21 \\
\text { Scores greater than or equal to } \\
11 \text { represent a case of anxiety or } \\
\text { depression, as applicable }\end{array}$ \\
\hline $\mathrm{PSQI}^{43} 44$ & $\begin{array}{l}\text { Index with } 18 \text { questions } \\
\text { assessing sleep quality and } \\
\text { disturbances during the previous } \\
\text { month. }\end{array}$ & $\begin{array}{l}\text { Subjective sleep quality; sleep } \\
\text { latency; duration of sleep; habitual } \\
\text { sleep efficiency; sleep disorders; use } \\
\text { of medications for sleep; daytime } \\
\text { dysfunction }\end{array}$ & $\begin{array}{l}\text { Range: } 0-21 \\
\text { Scores greater than } 5 \text { indicate } \\
\text { poor sleep quality }\end{array}$ \\
\hline QLQ-BR233940 & $\begin{array}{l}\text { Specific breast cancer scale with } \\
23 \text { questions assessing QoL in } \\
\text { patients with breast cancer } \\
\text { during the previous week and } \\
\text { month }\end{array}$ & $\begin{array}{l}\text { Functional scales: body image; } \\
\text { sexual functioning; sexual } \\
\text { enjoyment; future perspective } \\
\text { Symptom scales/items: systemic } \\
\text { therapy side effects; breast } \\
\text { symptoms; arm symptoms; concern } \\
\text { about hair loss }\end{array}$ & $\begin{array}{l}\text { Range (scales and single-item): } \\
0-100 \\
\text { Higher scores for a functional } \\
\text { scale represent a healthy level of } \\
\text { functioning. } \\
\text { Higher scores for a symptom } \\
\text { scale/item represent a higher } \\
\text { level of symptomatology/ } \\
\text { problems }\end{array}$ \\
\hline QLQ-C3039 41 & $\begin{array}{l}\text { Scale with } 30 \text { questions } \\
\text { assessing QoL in patients with } \\
\text { cancer during the previous week }\end{array}$ & $\begin{array}{l}\text { Global health status. } \\
\text { Functional scales: physical } \\
\text { functioning; role functioning; } \\
\text { emotional functioning; cognitive } \\
\text { functioning; social functioning. } \\
\text { Symptom scales/items: fatigue; } \\
\text { nausea and vomiting; pain; } \\
\text { dyspnoea; insomnia; appetite loss; } \\
\text { constipation; diarrhoea; financial } \\
\text { difficulties }\end{array}$ & $\begin{array}{l}\text { Range (scales and single-item): } \\
0-100 \\
\text { Higher scores for the global } \\
\text { health status and for a functional } \\
\text { scale represent a healthy level of } \\
\text { QoL and functioning, } \\
\text { respectively. } \\
\text { Higher scores for a symptom } \\
\text { scale/item represents a higher } \\
\text { level of symptomatology/ } \\
\text { problems }\end{array}$ \\
\hline $\mathrm{BPI}^{48} 49$ & $\begin{array}{l}\text { Questionnaire with } 9 \text { items used } \\
\text { to evaluate the severity of a } \\
\text { patient's pain and the impact of } \\
\text { this pain on the patient's daily } \\
\text { functioning in the past } 24 \mathrm{~h}\end{array}$ & $\begin{array}{l}\text { Severity of pain; impact of pain on } \\
\text { daily function; location of pain; pain } \\
\text { medications; amount of pain relief in } \\
\text { the past } 24 \mathrm{~h} \text { or the past week }\end{array}$ & $\begin{array}{l}\text { Range (for 'severity of pain' and } \\
\text { 'pain interference'): } 0-10 \\
\text { Higher scores for 'severity of } \\
\text { pain' and 'pain interference' } \\
\text { represent a higher level of pain } \\
\text { severity and pain interference, } \\
\text { respectively }\end{array}$ \\
\hline $\mathrm{DN} 4^{4748}$ & $\begin{array}{l}\text { Test with } 4 \text { questions ( } 10 \text { items) } \\
\text { for the screening of neuropathic } \\
\text { pain }\end{array}$ & Not applicable & $\begin{array}{l}\text { Range: } 0-10 \\
\text { Scores greater than or equal to } \\
4 \text { are regarded as indicative of } \\
\text { neuropathic pain }\end{array}$ \\
\hline $\mathrm{PDI}^{4850}$ & $\begin{array}{l}\text { Index with } 7 \text { items designed to } \\
\text { assess pain-related disability, } \\
\text { providing information that } \\
\text { complements assessment of } \\
\text { physical impairment }\end{array}$ & $\begin{array}{l}\text { Family/home responsibilities; } \\
\text { recreation; social activity; occupation; } \\
\text { sexual behaviour; self-care; } \\
\text { life-support activity }\end{array}$ & $\begin{array}{l}\text { Range: } 0-70 \\
\text { Higher scores represent greater } \\
\text { disability due to pain }\end{array}$ \\
\hline
\end{tabular}

BPI, Brief Pain Inventory; CIPN, chemotherapy-induced peripheral neuropathy; DN4, Neuropathic Pain Questionnaire; HADS, Hospital Anxiety and Depression Scale; MOCA, The Montreal Cognitive Assessment; PDI, Pain Disability Index; PSQI, Pittsburgh Sleep Quality Index; QLQ-BR23, Breast cancer-specific module of the Quality of Life Questionnaire of the European Organization for Research and Treatment of Cancer; QLQ-C30, Quality of Life Questionnaire of the European Organization for Research and Treatment of Cancer; QoL, quality of life.

Assembling of the main cohort and subcohorts and 1-year follow-up

Figure 2 describes the assembling of the main cohort and the NP and CIPN subcohorts. During 2012, all patients admitted to the IPO-Porto with a potential diagnosis of breast cancer were evaluated $(\mathrm{n}=961)$ and those who were proposed for surgical treatment and met the eligibility criteria were invited to participate $(n=588)$. 
961 potentially eligible patients admitted to the IPO-Porto (periods: 17.01.2012-17.08.2012 and 10.09.2012-18.12.2012)

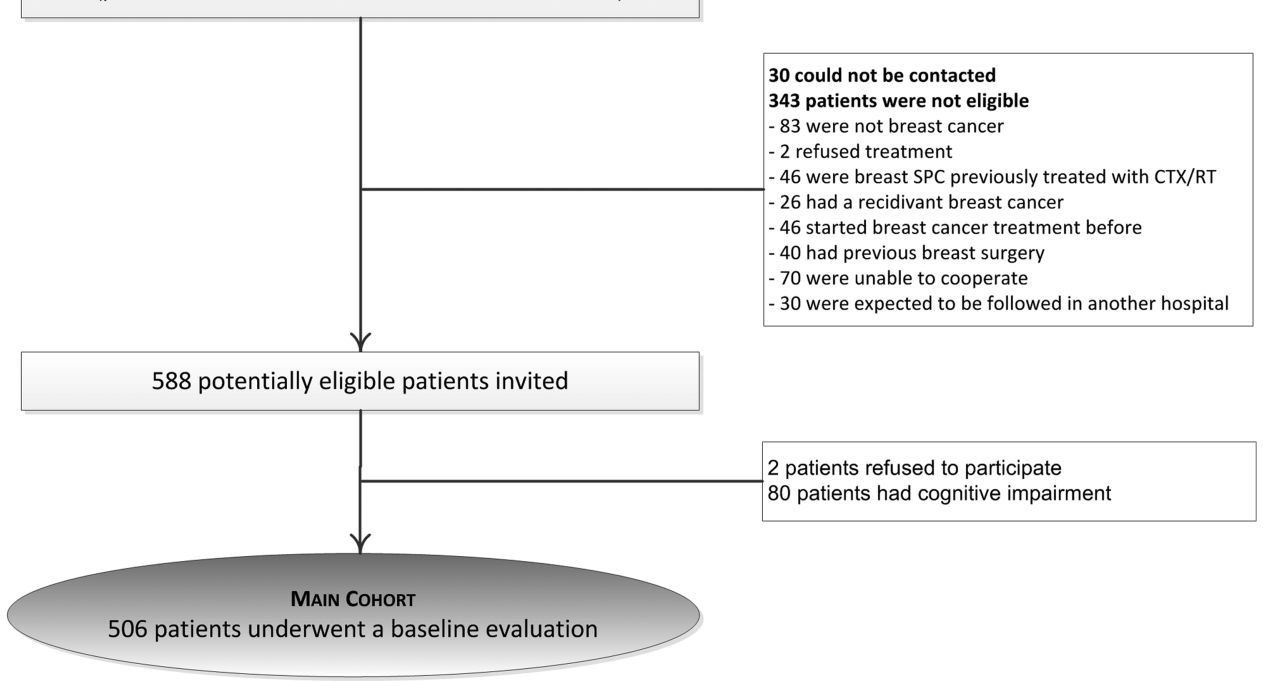

Figure 2 Flow chart describing the assembling of the main cohort and the neuropathic pain and chemotherapy-induced peripheral neuropathy subcohorts. CIPN, chemotherapy-induced peripheral neuropathy; CTX, chemotherapy; MoCA, The Montreal Cognitive Assessment; NP, neuropathic pain; RT, radiotherapy; SPC, second primary cancer.

Eighty patients with possible cognitive impairment were excluded and two refused to participate (no reason for refusing was specified). A total of 506 patients underwent a baseline evaluation before the first proposed treatment, constituting the main cohort. The subcohorts of NP and CIPN patients included those with a diagnosis of these conditions in the first year of follow-up.

The end of the follow-up period is scheduled for December 2015.

\section{ETHICS AND DISSEMINATION}

Written informed consent was obtained from all participants after the aims and procedures of the investigation had been fully explained by a member of the study group.

This is an observational investigation; as such we do not anticipate the occurrence of harmful effects related to participation in the study. To minimise the possible discomfort due to the need to go to the hospital for face-to-face evaluations or the duration of interviews, data collection procedures were designed to last no more than $60 \mathrm{~min}$, and are scheduled to take place on the same day as other appointments in the hospital as part of regular clinical care.

All data regarding clinical aspects are collected by clinical members of the research team and privacy is assured. We guarantee data protection in accordance with Portuguese law. Participants were coded with a unique non-identifying number; the correspondence between this code and the personal identifiable information is stored in a file, to which only the principal investigator can have access. Only the research team has access to the database with anonymised data, saved on a password-protected secure computer.
The expected results may contribute to a better understanding of the burden of neurological complications of breast cancer treatment and their role as mediators of the impact of the treatment in different dimensions of the patients' QoL. The main findings of the study will be submitted for publication in international peerreviewed journals and proposed for presentation at relevant international and national conferences. We will issue press releases to promote the dissemination of information relevant to the general population in the mass media. Moreover, this study will also contribute to the training of researchers through the production of master and doctoral theses.

Contributors NL and SP conceived and designed the study. SP and FF wrote the first version of the manuscript. NL, JC-L, TD and TS critically revised the manuscript for relevant intellectual content. All authors approved the final version for submission.

Funding The work of FF was supported by 'Fundação para a Ciência e a Tecnologia' (grant number SFRH/BD/92630/2013) and data management activities at baseline and 1-year follow-up were supported by the Chair on Pain Medicine of the Faculty of Medicine, University of Porto and by the Grünenthal Foundation - Portugal.

Competing interests None.

Ethics approval Ethics Committee of the Portuguese Institute of Oncology of Porto (Ref. CES 406/011 and CES 99/014).

Provenance and peer review Not commissioned; peer reviewed for ethical and funding approval prior to submission.

Open Access This is an Open Access article distributed in accordance with the Creative Commons Attribution Non Commercial (CC BY-NC 4.0) license, which permits others to distribute, remix, adapt, build upon this work noncommercially, and license their derivative works on different terms, provided the original work is properly cited and the use is non-commercial. See: http:// creativecommons.org/licenses/by-nc/4.0/ 


\section{REFERENCES}

1. Ferlay J, Soerjomataram I, Ervik M, et al. GLOBOCAN 2012 v1.0, Cancer incidence and mortality worldwide: IARC cancerbase No. 11. Lyon, France: International Agency for Research on Cancer, 2013. http://globocan.iarc.fr/ (accessed 16 Jul 2014).

2. Althuis MD, Dozier JM, Anderson WF, et al. Global trends in breast cancer incidence and mortality 1973-1997. Int J Epidemiol 2005;34:405-12.

3. Amaro J, Severo M, Vilela S, et al. Patterns of breast cancer mortality trends in Europe. Breast 2013;22:244-53.

4. Clarke M, Collins R, Darby S, et al. Effects of radiotherapy and of differences in the extent of surgery for early breast cancer on loca recurrence and 15-year survival: an overview of the randomised trials. Lancet 2005;366:2087-106.

5. Jatoi I, Miller AB. Why is breast-cancer mortality declining? Lancet Oncol 2003;4:251-54.

6. RORENO. Projeções da incidência de cancro na Região Norte2013, 2015 e 2020. Porto: Instituto Português de Oncologia do Porto, 2013.

7. Bastos J, Barros $\mathrm{H}$, Lunet N. [Breast cancer mortality trend in Portugal (1955-2002)]. Acta Med Port 2007;20:139-44.

8. Verdecchia A, Guzzinati S, Francisci S, et al. Survival trends in European cancer patients diagnosed from 1988 to 1999. Eur J Cancer 2009;45:1042-66.

9. Independent UK Panel on Breast Cancer Screening. The benefits and harms of breast cancer screening: an independent review. Lancet 2012;380:1778-86.

10. Soerjomataram I, Lortet-Tieulent J, Parkin DM, et al. Global burden of cancer in 2008: a systematic analysis of disability-adjusted life-years in 12 world regions. Lancet 2012;380:1840-50.

11. Howard-Anderson J, Ganz PA, Bower JE, et al. Quality of life, fertility concerns, and behavioral health outcomes in younger breast cancer survivors: a systematic review. J Natl Cancer Inst 2012;104:386-405.

12. Lemieux J, Goodwin PJ, Bordeleau LJ, et al. Quality-of-life measurement in randomized clinical trials in breast cancer: an updated systematic review (2001-2009). J Natl Cancer Inst 2011;103:178-231.

13. Montazeri A. Health-related quality of life in breast cancer patients: a bibliographic review of the literature from 1974 to 2007. J Exp Clin Cancer Res 2008;27:32.

14. Burstein HJ, Griggs JJ. Adjuvant hormonal therapy for early-stage breast cancer. Surg Oncol Clin N Am 2010;19:639-47.

15. Rocco N, Rispoli C, Pagano G, et al. Breast cancer surgery in elderly patients: postoperative complications and survival. BMC Surg 2013;13(Suppl 2):S25.

16. Pierce SM, Recht $\mathrm{A}$, Lingos $\mathrm{TI}$, et al. Long-term radiation complications following conservative surgery (CS) and radiation therapy (RT) in patients with early stage breast cancer. Int J Radiat Oncol Biol Phys 1992;23:915-23.

17. Azim HA Jr, de Azambuja E, Colozza M, et al. Long-term toxic effects of adjuvant chemotherapy in breast cancer. Ann Oncol 2011;22:1939-47.

18. Khasraw M, Posner JB. Neurological complications of systemic cancer. Lancet Neurol 2010;9:1214-27.

19. Giglio P, Gilbert MR. Neurologic complications of cancer and its treatment. Curr Oncol Rep 2010;12:50-9.

20. Jung BF, Ahrendt GM, Oaklander AL, et al. Neuropathic pain following breast cancer surgery: proposed classification and research update. Pain 2003;104:1-13.

21. Jung BF, Herrmann D, Griggs J, et al. Neuropathic pain associated with non-surgical treatment of breast cancer. Pain 2005;118:10-14.

22. Miltenburg NC, Boogerd W. Chemotherapy-induced neuropathy: a comprehensive survey. Cancer Treat Rev 2014;40:872-82.

23. Brouwers EE, Huitema AD, Boogerd W, et al. Persistent neuropathy after treatment with cisplatin and oxaliplatin. Acta Oncol 2009;48:832-41.

24. Smith EM, Beck SL, Cohen J. The total neuropathy score: a tool for measuring chemotherapy-induced peripheral neuropathy. Oncol Nurs Forum 2008;35:96-102.

25. Vincenzi B, Frezza AM, Schiavon G, et al. Identification of clinical predictive factors of oxaliplatin-induced chronic peripheral neuropathy in colorectal cancer patients treated with adjuvant Folfox IV. Support Care Cancer 2013;21:1313-19.

26. Bokhari FN, McMillan DE, McClement S, et al. Pilot study of a survey to identify the prevalence of and risk factors for chronic neuropathic pain following breast cancer surgery. Oncol Nurs Forum 2012;39:E141-9

27. Vilholm OJ, Cold S, Rasmussen L, et al. The postmastectomy pain syndrome: an epidemiological study on the prevalence of chronic pain after surgery for breast cancer. Br J Cancer 2008;99:604-10.
28. Peuckmann V, Ekholm O, Rasmussen NK, et al. Chronic pain and other sequelae in long-term breast cancer survivors: nationwide survey in Denmark. Eur J Pain 2009;13:478-85.

29. Gulluoglu BM, Cingi A, Cakir T, et al. Factors related to post-treatment chronic pain in breast cancer survivors: the interference of pain with life functions. Int J Fertil Womens Med 2006:51:75-82.

30. Edwards RR, Almeida DM, Klick B, et al. Duration of sleep contributes to next-day pain report in the general population. Pain 2008;137:202-7.

31. Raymond I, Nielsen TA, Lavigne G, et al. Quality of sleep and its daily relationship to pain intensity in hospitalized adult burn patients. Pain 2001;92:381-8.

32. Phyomaung PP, Dubowitz J, Cicuttini FM, et al. Are depression, anxiety and poor mental health risk factors for knee pain? A systematic review. BMC Musculoskelet Disord 2014;15:10.

33. Jensen MP, Chang HY, Lai YH, et al. Pain in long-term breast cancer survivors: frequency, severity, and impact. Pain Med 2010;11:1099-106.

34. Caffo $\mathrm{O}$, Amichetti M, Ferro $\mathrm{A}$, et al. Pain and quality of life after surgery for breast cancer. Breast Cancer Res Treat 2003;80:39-48.

35. Palesh OG, Collie K, Batiuchok D, et al. A longitudinal study of depression, pain, and stress as predictors of sleep disturbance among women with metastatic breast cancer. Biol Psychol 2007;75:37-44.

36. Nasreddine ZS, Phillips NA, Bedirian V, et al. The Montreal cognitive assessment, MoCA: a brief screening tool for mild cognitive impairment. J Am Geriatr Soc 2005;53:695-9.

37. Freitas S, Simoes MR, Alves L, et al. Montreal cognitive assessment (MoCA): normative study for the Portuguese population. J Clin Exp Neuropsychol 2011;33:989-96.

38. Freitas S, Simões MR, Martins C, et al. Estudos de adaptação do montreal cognitive assessment (MoCA) para a população portuguesa. Avaliação Psicológica 2010;9:345-57.

39. Aaronson NK, Ahmedzai S, Bergman B, et al. The European organization for research and treatment of cancer QLQ-C30: a quality-of-life instrument for use in international clinical trials in oncology. J Natl Cancer Inst 1993;85:365-76.

40. Sprangers MA, Groenvold M, Arraras JI, et al. The European organization for research and treatment of cancer breast cancer-specific quality-of-life questionnaire module: first results from a three-country field study. J Clin Oncol 1996;14:2756-68.

41. Pais-Ribeiro J, Pinto $C$, Santos $C$. Validation study of the Portuguese version of the QLC-C30-V.3. Psicologia, Saúde Doenças 2008;9:89-102.

42. Dewolf $\mathrm{L}$, Koller M, Velikova $\mathrm{G}$, et al. EORTC quality of life group translation procedure. 3rd edn. Brussels: EORTC Quality of Life Group Publication, 2009.

43. Buysse DJ, Reynolds CF III, Monk TH, et al. The Pittsburgh Sleep Quality Index: a new instrument for psychiatric practice and research. Psychiatry Res 1989;28:193-213.

44. Marques D, Gomes AA, Meiavia A, et al. Reliability and initial validation of the Pittsburgh Sleep Quality Index, European Portuguese version: a preliminary study in a sample of higher education students. Sleep Med. 2013;14,(Suppl 1):e140.

45. Pais-Ribeiro J, Silva I, Ferreira T, et al. Validation study of a Portuguese version of the hospital anxiety and depression scale. Psychol Health Med 2007;12:225-35.

46. Zigmond AS, Snaith RP. The hospital anxiety and depression scale. Acta Psychiatr Scand 1983;67:361-70.

47. Bouhassira $\mathrm{D}$, Attal $\mathrm{N}$, Alchaar $\mathrm{H}$, et al. Comparison of pain syndromes associated with nervous or somatic lesions and development of a new neuropathic pain diagnostic questionnaire (DN4). Pain 2005;114:29-36.

48. Azevedo LF, Pereira AC, Dias C, et al. [Translation, cultura adaptation and multicentric validation study of chronic pain screening and impact assessment instruments]. Dor 2007;15:6-56.

49. Cleeland CS, Ryan KM. Pain assessment: global use of the brief pain inventory. Ann Acad Med Singapore 1994;23:129-38.

50. Pollard CA. Preliminary validity study of the pain disability index. Percept Mot Skills 1984;59:974.

51. Edge SB, Byrd DR, Compton CC, et al. AJCC cancer staging manual. 7th edn. New York: Springer; 2010.

52. CTCAE. Cancer Therapy Evaluation Program, Common Terminology Criteria for Adverse Events, Version 4.0. DCTD, NCI, NIH, DHHS, 2010. http://ctep.cancer.gov/ (accessed 1 Jan 2012).

53. Osoba D, Rodrigues G, Myles J, et al. Interpreting the significance of changes in health-related quality-of-life scores. J Clin Oncol 1998;16:139-44.

54. RORENO. Análise de Sobrevivência, Principais Cancros da Região Norte, 2000/2001. Porto: Registo Oncológico Regional do Norte, 2010. 